\title{
Venous Thromboembolism Therapy with Apixaban in Daily Care Patients: Results from the Dresden NOAC Registry
}

\author{
Jan Beyer-Westendorf ${ }^{1,2}$ Sandra Marten ${ }^{1} \quad$ Luise Tittl $^{1} \quad$ Christiane Naue $^{1} \quad$ Martin Bornhäuser $^{1}$ \\ ${ }^{1}$ Thrombosis Research Unit, Division Hematology, Department of \\ Address for correspondence Jan Beyer-Westendorf, MD, Division \\ Medicine I, University Hospital “Carl Gustav Carus” Dresden, \\ Dresden, Germany \\ 2 Kings Thrombosis Service, Department of Hematology, \\ Kings College, London, United Kingdom \\ Haematology, Department of "Thrombosis Research of Medicine I, \\ University Hospital “Carl Gustav Carus," Technical University Dresden, \\ Fetscherstrasse 74, Dresden 01307, Germany \\ (e-mail: jan.beyer@uniklinikum-dresden.de).
}

TH Open 2021;5:e143-e151.

\begin{abstract}
The effectiveness and safety of venous thromboembolism (VTE) treatment with apixaban, demonstrated in phase III trials, need to be confirmed in daily care.

Using data from the prospective, noninterventional cross-indication Dresden NOAC Registry we evaluated rates of VTE recurrence and bleeding complications during apixaban treatment of VTE patients. For this analysis, we only included patients with acute VTE who started apixaban within 14 days after diagnosis and who were enrolled within these 14 days. Patient characteristics, treatment persistence, and clinical outcomes were assessed.

Between August 1st, 2014 and October 31, 2018, 352 patients with apixaban treatment for acute VTE were enrolled. During treatment (median exposure $13.7 \pm 9.8$ months; median follow-up $21.7 \pm 6.1$ months) rates of recurrent VTE and International Society on Thrombosis and Haemostasis major bleeding were 1.3/100 pt. years (95\% confidence interval or $\mathrm{Cl} 0.4-3.0$ ) and $1.5 / 100$ pt.years (0.6-3.3), respectively. At 6 months. $68.6 \%$ of patients were still taking apixaban, $23.9 \%$ had a scheduled end of treatment, $6.3 \%$ were switched to other anticoagulants, and the remaining $2.3 \%$

Keywords

- anticoagulation

- bleeding

- persistence

- apixaban

- venous thromboembolism

had unplanned complete discontinuation of anticoagulation.

Of the 188 patients stopping apixaban, 12 (6.4\%) experienced a recurrent VTE (six pulmonary embolisms \pm deep vein thrombosis, six deep vein thrombosis; mean time between stopping anticoagulation and VTE recurrence $5.2 \pm 4.1$ months [range 14-417 days]).

Our findings suggest that, in daily care, apixaban demonstrated high effectiveness, safety, and persistence in the treatment of acute VTE with low rates of unplanned discontinuation.
\end{abstract}

\section{Introduction}

After decades of VTE therapy with vitamin $\mathrm{K}$ antagonists (VKAs), nonvitamin $\mathrm{K}$ antagonizing oral anticoagulants (NOACs) have replaced VKA in this indication, following successful completion of large phase III trials some 10 years

received

April 23, 2020

accepted after revision

February 23, 2021
DOI https://doi.org/

10.1055/s-0041-1728675.

ISSN 2512-9465. ago. ${ }^{1}$ The main benefits of NOAC include a much more predictable dose-response relationship, limited interactions with co-medications and, therefore, a better convenience for patients who no longer require routine monitoring or frequent dose adjustments. ${ }^{2,3}$
(C) 2021. The Author(s).

This is an open access article published by Thieme under the terms of the Creative Commons Attribution License, permitting unrestricted use, distribution, and reproduction so long as the original work is properly cited. (https://creativecommons.org/licenses/by/4.0/)

Georg Thieme Verlag KG, Rüdigerstraße 14, 70469 Stuttgart, Germany 
Apixaban is a direct factor Xa inhibitor that has been approved for the treatment of deep vein thrombosis (DVT) and pulmonary embolism (PE), following the finalization of two large phase III trials (AMPLIFY and AMPLIFY EXTENSION) ${ }^{4,5}$ and, more recently, the cancer VTE study CARAVAGGIO. ${ }^{6}$

In AMPLIFY, apixaban was tested against low molecular weight heparin (LMWH) followed by VKA for the treatment of acute DVT and/or PE. Of note, in this study only patients with unprovoked VTE or provoked VTE with persisting risk factors were included and patients were excluded if they received other anticoagulants for initial VTE therapy for more than 36 hours. In this study, apixaban demonstrated noninferiority in efficacy and superiority for safety in comparison to $\mathrm{LMWH} / \mathrm{VKA}$.

In the three-armed AMPLIFY EXTENSION trial, two doses of apixaban (continued therapeutic dosage of $5 \mathrm{mg}$ BID vs. prophylactic dosage of $2.5 \mathrm{mg}$ BID) were compared against placebo in VTE patients who had completed a 6 to 12 months course of anticoagulation for acute DVT and/or PE and in whom uncertainty existed whether anticoagulation should be continued or stopped at this point in time. In this trial, both apixaban dosages drastically reduced the risk of recurrent VTE (relative risk reduction 64 and 67\% compared with placebo) without increasing the risk of major bleeding.

Based on these results, apixaban has been approved and rapidly adopted in many countries and current guidelines list apixaban as one of the preferred options for acute and longterm VTE treatment. ${ }^{2,3}$

However, as with all phase III trials, the question of external validity arises, since these trials apply rigorous inclusion and exclusion criteria, regulate all medical interventions around the target disease, and apply close monitoring and strict outcome assessments, ensuring maximal patient safety and data quality. ${ }^{7}$ At the same time, these prespecifications may limit generalizability of trial data to the daily care management of patients with acute or chronic VTE. Therefore, observational studies are requested by physicians and authorities to complement phase III trial findings. Furthermore, such observational studies can supplement phase III trial data by evaluating patient selection patterns, outcome management, and treatment persistence to anticoagulation, all of which is difficult to evaluate in a randomized setting that applies strict prespecified management recommendations.

With this in mind, we set up the prospective multicentric cross-indication DRESDEN NOAC REGISTRY in 2011, which is still ongoing. Here, we report the collected data on acute VTE management with apixaban.

\section{Methods}

\section{Patients}

The DRESDEN NOAC REGISTRY (NCT01588119) is a prospective registry in the administrative district of Dresden (Saxony), Germany, covering a network of more than 230 enrolling physicians from private practices and hospitals. Patients are eligible if they receive at least 3 months of NOAC anticoagulation for any indication and are willing to participate in and available for quarterly follow-up visits by phone, performed by the central registry office. No exclusion criteria apply. The design and methodology of the Dresden NOAC Registry has been published previously. ${ }^{8}$

As a noninterventional study, patients are not actively examined for recurrence. In case of suspected outcome events, medical reports are requested from health care providers and presented to an adjudication committee. If medical reports are inconclusive, the worst-case approach is applied.

The protocol does not include a formal adjudication of the index DVT/PE, since diagnosis of acute VTE in Germany is predominantly in the hands of vascular or cardiac specialists and objective testing (mainly with ultrasound for suspected DVT and computed tomography pulmonary angiography or $\mathrm{V} / \mathrm{Q}$ scan for suspected PE) is readily available. The established standard of care is consistently used across Germany, as has been demonstrated by the TULIPA registry. ${ }^{9}$

For the presented analysis, only patients with acute PE and/or acute distal or proximal lower limb DVT who started apixaban within 14 days after diagnosis of VTE and who were enrolled within these 14 days were evaluated with regard to patient characteristics, treatment persistence, and clinical outcomes.

\section{Outcome Measures}

To assess effectiveness of apixaban therapy in VTE, the annualized rate of the recurrent VTE was evaluated. Cases of sudden death were adjudicated as fatal PE and counted as a recurrent VTE event if other causes of death were not established, and PE could not be ruled out.

The main safety outcome was the annualized rate of major bleeding according to the International Society on Thrombosis and Haemostasis (ISTH) definition. ${ }^{10}$ Further safety outcomes were rates of ISTH nonmajor clinically relevant (NMCR) bleeding, minor bleeding, and all-cause mortality.

Crude outcome numbers are reported for days 90, 180, 365, and $>365$ and annualized event rates for 180 and 365 days.

To put outcome event rates into perspective with available real-world data in the discussion section, a formal literature search was performed on February 15, 2020 using the search terms "apixaban" in combination with "deep vein thrombosis or DVT," "pulmonary embolism or PE," "venous thromboembolism or VTE," and "real world."

\section{Treatment Discontinuation}

In accordance with previously published analyses from our registry, ${ }^{11}$ treatment discontinuation was defined as a permanent discontinuation or an unscheduled interruption of apixaban for longer than 4 weeks without the initial plan to restart apixaban. This included patients who were permanently switched to another anticoagulant. In contrast, treatment persistence was defined as the continuation of apixaban therapy over the entire follow-up period, allowing for temporary interruptions. At every visit, any change in anticoagulant therapy is assessed. Reasons for switching to other anticoagulants or stopping anticoagulation as well as the future treatment plan were obtained from patients or attending physicians. Missing values were left blank and not replaced by imputation. 


\section{Statistics}

Two different analysis sets were defined and evaluated:

1. The overall rate of recurrent VTE was evaluated in the intention-to-treat analysis, including all VTE patients who were enrolled in the registry and received apixaban for acute VTE at baseline. In this analysis, all effectiveness outcome events were included that occurred throughout the follow-up period, despite the anticoagulation status.

2. Rates of recurrent VTE and bleeding complication (all, major, and NMCR) during the time of anticoagulation therapy (and up to 3 days after last intake) were evaluated in the on-treatment analysis. Temporary treatment interruptions (up to 3 days) were tolerated.

Data analysis was conducted according to a predefined statistical analysis plan. Baseline characteristics are presented as absolute and relative frequencies, mean and standard deviation, or median with interquartile range, where appropriate. Annualized event rates (per 100 patient years) were calculated using Kaplan-Meier time-to-first-event analysis and reported with the corresponding 95\% confidence intervals (CIs). Due to the small sample size, comparative statistics of subgroups were omitted.

In addition, the following sensitivity analyses were performed as Kaplan-Meier time-to-first event analyses:

- Recurrent VTE during the acute phase until day 90 for patients who started apixaban within 72 hours; 3 to 7 days or 8 to 14 days after VTE diagnosis.

- Recurrent VTE during the acute phase until day 90 for patients who were treated for provoked VTE by major transient trigger versus minor transient or persistent trigger versus unprovoked VTE. Major transient triggers included complete immobilization for at least 3 days or major surgery within 4 weeks prior to VTE diagnosis or active cancer. Surgery was deemed "major" if general anesthesia for greater than 30 minutes was performed. ${ }^{12}$ Minor triggers included long-distance travel, acute infectious diseases without immobilization, estrogen use, obesity with BMI $>30$, pregnancy or puerperium, family history of VTE.

- Net clinical benefit was observed (defined as recurrent VTE and/or major bleeding and/or all-cause mortality) for patients who had a scheduled end of treatment at 6 months and stopped apixaban between days 150 and 210 versus those who were selected to continue apixaban beyond 210 days (who, in case of later treatment cessation, were censored at the day of apixaban discontinuation).

All statistical analyses were performed using the IBM SPSS Statistics version 25, MedCalc version 14.8.1.

\section{Results}

Between December 1st, 2011 and October 31, 2018, a total of 4,385 patients were enrolled into the DRESDEN NOAC REGISTRY. Of these, 469 were receiving apixaban for VTE treatment and 352 (75.1\%) fulfilled the selection criteria for the present analysis. Reasons for exclusion are demonstrated in -Fig. $\mathbf{1}$.

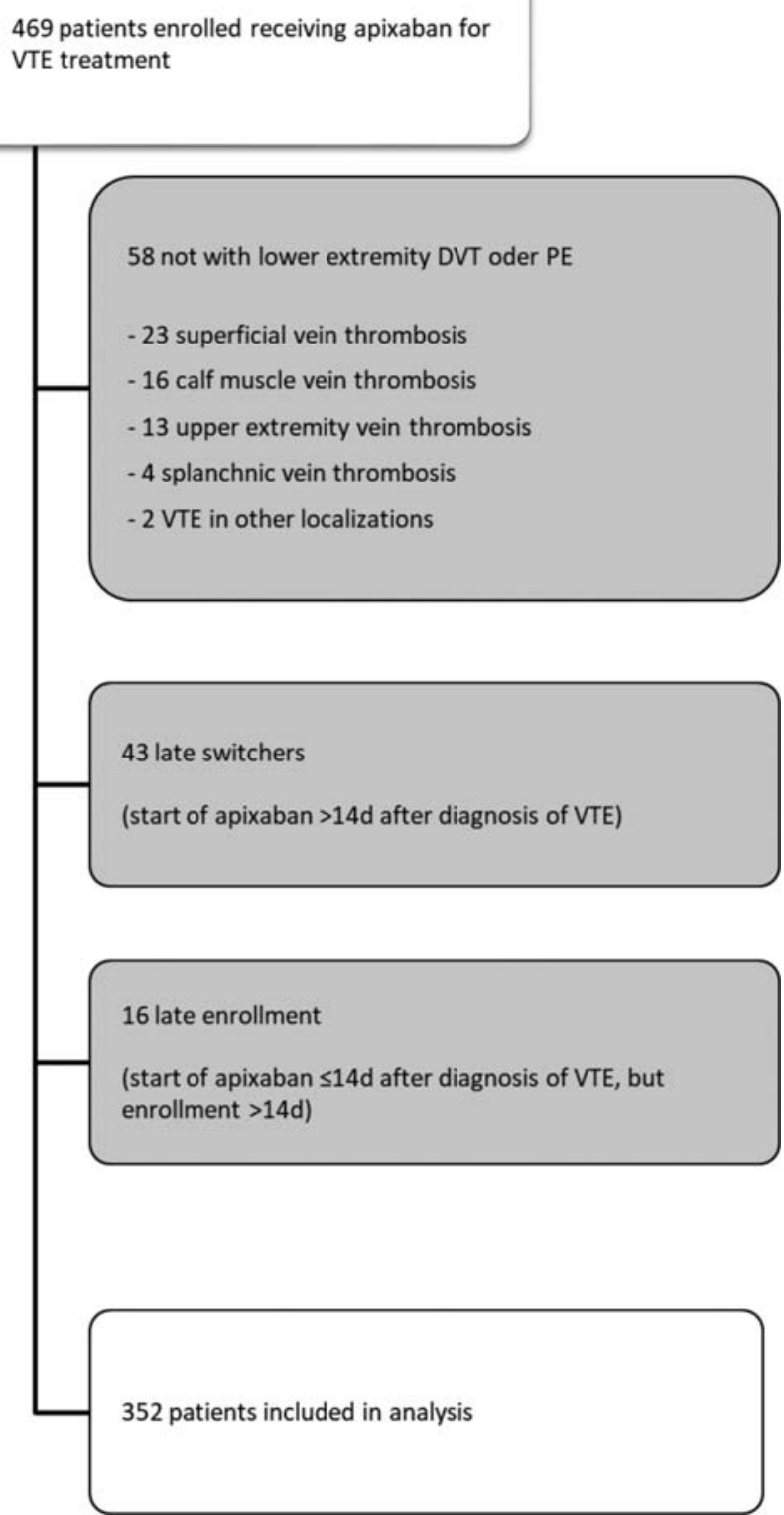

Fig. 1 Flowchart of study cohort.

The VTE index event was an isolated DVT without confirmed PE in 276 (78.4\%) cases and 76 (21.6\%) patients had an objectively confirmed PE with or without DVT. Overall, 51.4\% were male and median age was 66 years (IQR 25 years). Details on patient characteristics and index VTE are presented in - Table 1. Mean time between VTE diagnosis and initiation of apixaban was $1.0 \pm 2.7$ days (median 0 days; IQR 0 days) and numerically longer for PE versus DVT (mean $2.9 \pm 3.8$ days vs. $0.5 \pm 1.9$ days). At baseline, apixaban was prescribed at a dose of $10 \mathrm{mg}$ BID in $90.9 \%, 5 \mathrm{mg}$ BID in $7.1 \%$, and $2.5 \mathrm{mg}$ BID in $2.0 \%$ of patients. Reasons for not using $10 \mathrm{mg}$ apixaban BID initially were pretreatment with therapeutic parenteral anticoagulants for $\geq 7$ days in 20 cases, comorbidities (e.g., bleeding history, renal impairment) in seven cases, and not reported in the remaining five cases.

During a mean follow-up (FU) of $21.7 \pm 6.1$ months (median 24 months; IQR 5.2; range 31-1,114 days), a total of 17 patients 
Table 1 Patient characteristics at baseline

\begin{tabular}{|c|c|c|c|}
\hline & $\begin{array}{l}\text { All } \\
n=352\end{array}$ & $\begin{array}{l}\text { DVT } \\
n=276\end{array}$ & $\begin{array}{l}\mathrm{PE} \pm \mathrm{DVT} \\
n=76\end{array}$ \\
\hline Male, $n(\%)$ & $181(51.4)$ & $136(49.3)$ & $45(59.2)$ \\
\hline Median age (IQR), years & $66.0(25.0)$ & $66.5(25.0)$ & $65.5(23.0)$ \\
\hline Number of concomitant drugs, mean (SD) & $3.0 \pm 3.0$ & $2.8 \pm 2.9$ & $4.1 \pm 3.2$ \\
\hline $\begin{array}{l}\text { Mean time between VTE diagnosis and } \\
\text { initiation of apixaban (SD), days }\end{array}$ & $1.0 \pm 2.7$ & $0.5 \pm 1.9$ & $2.9 \pm 3.8$ \\
\hline Unprovoked VTE, n (\%) & $118(33.5)$ & $87(31.5)$ & $31(40.8)$ \\
\hline $\begin{array}{l}\text { Event VTE provoked by minor persistent } \\
\text { or transient triggers, } n(\%)\end{array}$ & $173(49.1)$ & $136(49.3)$ & $37(48.7)$ \\
\hline Event VTE provoked by major transient triggers, $n$ (\%) & $61(17.3)$ & $53(19.2)$ & $8(10.5)$ \\
\hline Recurrent VTE, $n(\%)$ & $82(23.3)$ & $65(23.6)$ & $17(22.4)$ \\
\hline Proximal vs. distal DVT, $n(\%)$ & 197 (56.0) vs. $79(22.4)$ & $197(71.4)$ vs. $79(28.6)$ & n.a. \\
\hline Malignant disease, $n(\%)$ & $42(11.9)$ & $33(12.0)$ & $9(11.8)$ \\
\hline - Active cancer, $n(\%)$ & $5(1.4)$ & $1(0.4)$ & $4(5.3)$ \\
\hline Glomerular filtration rate (GFR) $<50 \mathrm{~mL} / \mathrm{min}, n(\%)$ & $31(8.8)$ & $23(8.3)$ & $8(10.5)$ \\
\hline - GFR 30-50 mL/min, $n(\%)$ & $29(8.2)$ & $21(7.6)$ & $8(10.5)$ \\
\hline - GFR <30 mL/min, $n$ (\%) & $2(0.6)$ & $2(0.7)$ & 0 \\
\hline
\end{tabular}

Abbreviations: DVT, deep vein thrombosis; GFR, glomerular filtration rate; IQR, interquartile range; PE, pulmonary embolism; SD, standard deviation; VTE, venous thromboembolism.

(4.8\%) experienced a recurrent VTE, which translated into a recurrence rate of 2.8/100 patient years (pt. years; $95 \% \mathrm{CI}$ 1.6-4.4) for the intention-to-treat population.

The mean time of apixaban exposure was $13.7 \pm 9.8$ months (median 12.4 months; IQR 20.4) and, during active treatment with apixaban, five patients experienced a recurrent VTE. This translated into a recurrence rate of $1.3 / 100 \mathrm{pt}$. years (95\% CI 0.4-3.0) for the on-treatment population. VTE recurrence rates were highest during the first 90 days of therapy ( - Table 2; - Supplementary Fig. S1).

A total of 124 patients (35.2\%; $43 / 100$ pt. years; $95 \% \mathrm{Cl}$ 35.7-51.2) reported any bleeding events during apixaban exposure. ISTH major bleeding occurred in six cases $(1.5 / 100$ pt. years; 95\% CI 0.6-3.3), including three intracranial bleedings, two cases of genitourinary bleeding, and one case of postoperative bleeding with drop in hemoglobin $>2 \mathrm{~g} / \mathrm{dL}$. Furthermore, NMCR bleeding events occurred in 55 cases (15.6\%; 15.3/100 pt. years; $95 \%$ CI 11.6-20.0), predominantly manifesting as skin/mucosal bleeding (61.8\%), genitourinary bleeding (20.0\%), gastrointestinal bleeding (12.7\%), or other bleeding types (5.5\%).

During apixaban treatment, rates for recurrent VTE were numerically lower in patients with DVT as an index event (1.1/100 pt. years; $95 \% \mathrm{CI} 0.2-3.1$ ) compared with PE as an index event (1.8/100 pt. years; 95\% CI 0.2-6.5) and similar trends were observed for rates of major bleeding (1.1/100 pt. years for index DVT; $95 \%$ Cl $0.2-3.1$ vs. 2.7/100 pt. years for index PE; 95\% Cl 0.6-7.8). Effectiveness and safety profiles were consistent across relevant subgroups (- Supplementary Table S1 and -Supplementary Fig. S2). Furthermore, in another sensitivity analysis we did not detect a signal that early (within 2 days after VTE diagnoses) or delayed (8-14 days after diagnosis) start of apixaban therapy correlated to an increased risk of VTE recurrence (-Supplementary Fig. S3).

A total of $12 / 352$ patients died (1.9/100 pt. years; $95 \% \mathrm{CI}$ 1.0-3.3) of which one death occurred during or within 3 days after last intake of apixaban $(0.25 / 100$ pt. years; $95 \% \mathrm{CI}$ $0-1.4)$. Causes of death were cancer $(n=5)$, fatal

Table 2 Outcome event rates according to treatment phase and treatment continuation

\begin{tabular}{|c|c|c|c|c|c|c|c|c|}
\hline \multirow[t]{2}{*}{$n=352$} & \multicolumn{2}{|c|}{ Events $0-90 \mathrm{~d}$} & \multicolumn{2}{|c|}{ Events $91-180 \mathrm{~d}$} & \multicolumn{2}{|c|}{ Events $181-365 \mathrm{~d}$} & \multicolumn{2}{|c|}{ Events $>365 \mathrm{~d}$} \\
\hline & ITT & On treatment & ITT & On treatment & ITT & On treatment & ITT & On treatment \\
\hline Recurrent VTE; $n$ (\%) & $3(0.9)$ & $2(0.6)$ & $1(0.3)$ & 0 & $5(1.4)$ & 0 & $8(2.3)$ & $3(0.9)$ \\
\hline Fatal VTE; $n(\%)$ & 0 & 0 & 0 & 0 & 0 & 0 & 0 & 0 \\
\hline Major bleeding; $n$ (\%) & $3(0.9)$ & 0 & $2(0.6)$ & 0 & $4(1.1)$ & $3(0.9)$ & $6(1.7)$ & $3(0.9)$ \\
\hline Fatal bleeding; $n$ (\%) & 0 & 0 & $1(0.3)$ & 0 & 0 & 0 & 0 & 0 \\
\hline Mortality; n (\%) & $1(0.3)$ & 0 & $3(0.9)$ & 0 & $2(0.6)$ & 0 & $6(1.7)$ & $1(0.3)$ \\
\hline
\end{tabular}

Abbreviations: ITT, intention-to-treat population, which includes all outcome events during follow-up, irrespective of anticoagulation status; VTE, venous thromboembolism. 
cardiovascular event ( $n=2$ including one case of sudden cardiac death and one case of congestive heart failure), sepsis/infection $(n=2)$, age-related death $(n=2)$, and one case of fatal bleeding unrelated to apixaban exposure.

At 6 months (FU completed in 352 pts.), 341 patients (96.9\%) were still alive. Of these, $69.8 \%$ of patients were still taking apixaban, $24.6 \%$ had a scheduled end of treatment, $6.5 \%$ were switched to other anticoagulants, and the remaining $2.3 \%$ had unplanned complete discontinuation of anticoagulation.

At 12 months (FU completed in 328 pts.), 316 patients (96.3\%) were still alive. Of these, $57.9 \%$ of patients were still taking apixaban. The remaining patients having a scheduled end of treatment (33.5\%), were switched to other anticoagulants $(8.5 \%)$ or had an unplanned complete discontinuation of anticoagulation (3.8\%).

Of the 188 patients stopping apixaban, 12 (6.4\%) experienced a recurrent VTE (six PE \pm DVT, six DVT) and the mean time between stopping anticoagulation and VTE recurrence was $5.2 \pm 4.1$ months. Despite discontinuation of oral anticoagulation in a total of 188 patients, major bleeding still occurred in nine patients (4.8\%; - Supplementary Table S2), which translated into an anticoagulation-unrelated major bleeding rate of 3.9/100 pt. years (95\% CI 1.8-7.5), indicating that the major driver for bleeding is patient-related and not anticoagulation-related, which likely contributed to the decision to stop anticoagulation.

\section{Discussion}

In a large cohort of patients treated with apixaban for acute VTE in daily care, we could demonstrate a general confirmation of the effectiveness and safety of apixaban demonstrated in phase III studies. In the AMPLIFY phase III trial which did not report annualized rates, absolute rates of recurrent VTE and ISTH major bleeding were 2.3 and $0.6 \%$, respectively for patients treated for 6 months. ${ }^{4,5}$ In our intention-to-treat analysis, recurrent VTE occurred in $4.8 \%$ of patients $(2.8 / 100$ pt. years) and ISTH major bleeding in $4.3 \%$ of patients (2.4/100 pt.years). The higher event rates seen in our study may partly be explained by the fact that no exclusion criteria were applied in our cohort of consecutive daily care patients, whereas phase III trials prohibit enrolment of patients at unacceptably high risk for complications by defining explicit inclusion and exclusion criteria. Furthermore, mean age of our cohort (66 years) was considerably higher than that in the AMPLIFY (mean 57 years). ${ }^{4}$ In addition, the majority of patients in AMPLIFY were patients with unprovoked VTE (excluding patients with VTE shortly after major trauma or surgery, which may carry a higher risk for VTE recurrence or bleeding especially in the early phase of anticoagulation), whereas we included a total of 61 (17.3\%) patients with VTE provoked by a major transient risk factor such as recent surgery or trauma. Numerically, these patients presented slightly higher rates of VTE recurrence ( 1.6 vs. $0.8 \%$ in patients with unprovoked VTE; - Supplementary Table S1) but the absolute difference was small and based on low numbers. Reassuringly, rates for major bleeding were low in both cohorts. Another difference between AMPLIFY and our cohorts' study was the length of observation (6 vs. 21 months). Therefore, neither our crude bleeding incidences nor our annualized bleeding event rates, derived from a cohort with considerably longer treatment and follow-up duration, can be directly compared with the phase III data. However, at 6 months, our crude event rates of 1.1\% (recurrent VTE) and $1.4 \%$ (major bleeding; - Table 2 ) were not far from those reported in AMPLIFY.

In addition to thromboembolic and bleeding outcomes we reported on treatment persistence and reasons for discontinuation. We found a reassuringly low rate of unscheduled premature discontinuation ( $2.3 \%$ at 6 and $3.7 \%$ at 12 months) and a clear trend toward prolonged anticoagulant treatment with apixaban, since nearly $70 \%$ of patients were treated for longer than 6 months. The low rate of unscheduled premature discontinuation is reassuring and an indicator of high patient and physician satisfaction. The trend to prolonged treatment is in line with the pronounced risk profile for VTE recurrence in our cohort and, thus, in line with current guideline recommendations.

Our data are not the first real-life data for VTE treatment with apixaban. Several large retrospective health record or claims data analyses from Denmark and the United States have been published. ${ }^{13-17}$ Although all these studies have evaluated much larger cohorts of VTE patients, by definition they are retrospective in nature, mostly rely on ICD codes, and apply algorithms to identify potential outcome events which are not centrally adjudicated. As such, there are many known and unknown confounders in these studies, which also lack granularity in details of patient selection, drug management, and reasons for treatment changes. For this, prospective registry data are needed and, to our knowledge, only one such prospective registry has been published do date. ${ }^{18}$ In this prospective single center cohort of 300 apixaban patients, only those who completed 3 months of treatment or had an outcome event were evaluated, resulting in a total of 302 patients. Rates of recurrent event at 3 months was $2.3 \%$ and major bleeding occurred in 11 patients (3.6\%), which is somewhat higher than our data, although no direct comparisons can be made, since treatment durations were different and no details on drug dosage or exposure time were reported. It has also to be noted that in this Mayo Clinic project, a total of 1,696 consecutive patients were enrolled, of which only $17.8 \%$ received apixaban, which indicates a high degree of dedicated patient selection. Strikingly, this group reported surprisingly low rates of NMCR bleeding (2.3\%) which is not in line with our data $(15.6 \%)$ or those collected in AMPLIFY, raising the question for a potential of underreporting of nonmajor bleeding events in their study.

In contrast, our study reports on a cohort of apixaban patients recruited at multiple centers and we followed all patients (irrespective of premature treatment discontinuation) for up to 2.5 years. We reported outcome events that occurred during active treatment and after discontinuation and provided outcome data based on central event adjudication. Our data also did not indicate that early start of apixaban or prolonged alternative (usually LMWH) therapy 


\begin{tabular}{|c|c|c|c|c|c|c|c|c|}
\hline 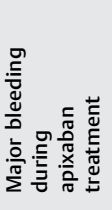 & 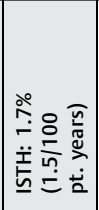 & 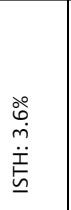 & 兰 & 兰 & 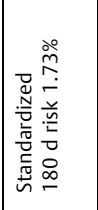 & 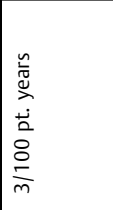 & 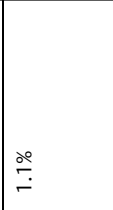 & 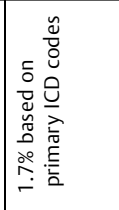 \\
\hline 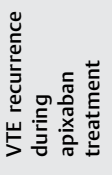 & 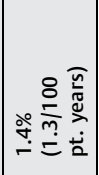 & $\stackrel{\circ}{\stackrel{一}{N}}$ & 亭 & $\breve{5}$ & 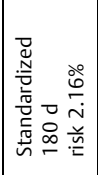 & 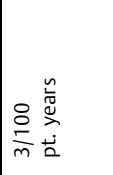 & @ִ & $\stackrel{\circ}{\stackrel{\sim}{~}}$ \\
\hline 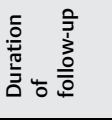 & $\begin{array}{l}\stackrel{̊}{E} \\
\hat{\sim} \\
\dot{N}\end{array}$ & $\underset{m}{\stackrel{O}{E}}$ & 兰 & $\breve{5}$ & 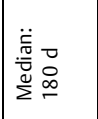 & $\begin{array}{l}\text { ठ } \\
\text { ğ }\end{array}$ & 兰 & 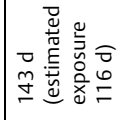 \\
\hline 总 & 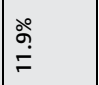 & $\begin{array}{l}\stackrel{\circ}{\circ} \\
\dot{\sigma}\end{array}$ & 兰 & $\breve{\breve{s}}$ & 守 & 童 & 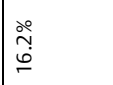 & ְ̊ \\
\hline 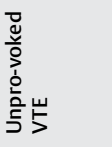 & 总 & 递 & 兰 & $\breve{5}$ & 弚 & $\begin{array}{l}\text { ه̊ำ } \\
\text { ஸे }\end{array}$ & 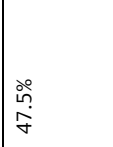 & 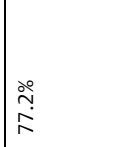 \\
\hline 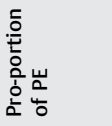 & $\stackrel{\stackrel{\leftrightarrow}{\sim}}{\stackrel{\sim}{N}}$ & 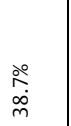 & 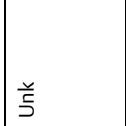 & $\breve{5}$ & 总 & す̊̊ & 羌 & \begin{tabular}{|l} 
号 \\
$\dot{\sigma}$ \\
$\dot{\sigma}$
\end{tabular} \\
\hline 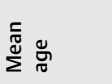 & $\vec{b}$ & 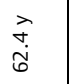 & 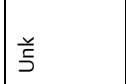 & 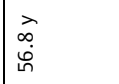 & 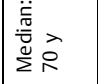 & \begin{tabular}{|l}
$\overrightarrow{0}$ \\
$\dot{0}$ \\
$\dot{0}$
\end{tabular} & $\begin{array}{l}\text { aे } \\
\text { के } \\
\text { ஸे }\end{array}$ & $\begin{array}{l}a \\
\dot{0} \\
\dot{0}\end{array}$ \\
\hline 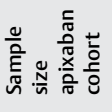 & 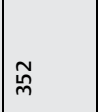 & స్ & $\mid$\begin{tabular}{l}
0 \\
\multirow{2}{0}{} \\
$i$
\end{tabular} & $\underset{\substack{\infty \\
\sim \\
\sim \\
f}}{ }$ & 苦 & g. & \begin{tabular}{|l} 
\\
0 \\
0 \\
$\infty$
\end{tabular} & $\mid$ \\
\hline 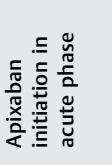 & $\stackrel{\check{\nu}}{\check{\nu}}$ & $\stackrel{\check{\nu}}{\searrow}$ & 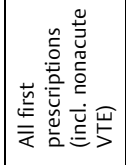 & 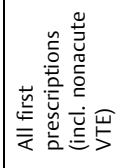 & $\stackrel{\check{0}}{\rightleftharpoons}$ & 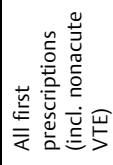 & 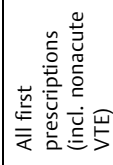 & 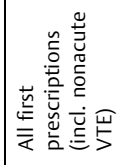 \\
\hline 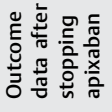 & 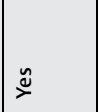 & $\theta$ & $\theta$ & $\theta$ & $\theta$ & $\theta$ & $\theta$ & a \\
\hline 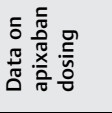 & $\stackrel{\breve{\nu}}{\check{\nu}}$ & Q & $\stackrel{\check{\varpi}}{\rightleftharpoons}$ & $\theta$ & $\theta$ & Q & Q & Q \\
\hline 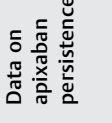 & $\stackrel{\check{\nu}}{\check{\nu}}$ & 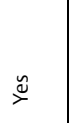 & Q & $\theta$ & $\theta$ & $\theta$ & $\theta$ & Q \\
\hline 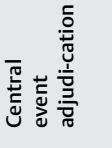 & $\stackrel{\check{\nu}}{\check{\nu}}$ & 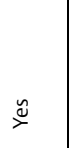 & Q & a & $\theta$ & Q & Q & Q \\
\hline 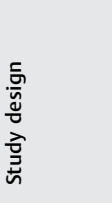 & 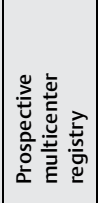 & 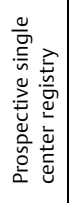 & 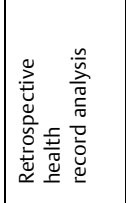 & 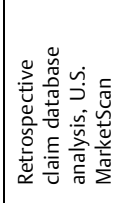 & 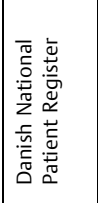 & 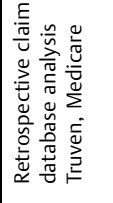 & 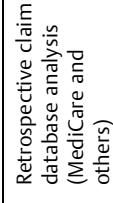 & 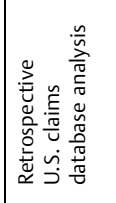 \\
\hline 离 & 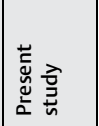 & 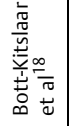 & 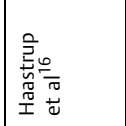 & 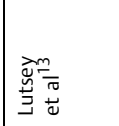 & 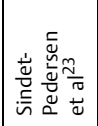 & 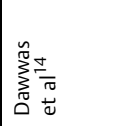 & 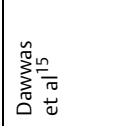 & 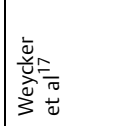 \\
\hline
\end{tabular}


for up to 2 weeks before apixaban initiation leads to different rates in VTE recurrence, which is also reassuring, since AMPLIFY did not allow for a pretreatment with LMWH for more than 36 hours. Furthermore, we assessed treatment persistence and reasons for treatment changes, all of which have not been reported so far. To put our findings into perspective with the abovementioned reports we have summarized the main design items and results of published apixaban VTE cohorts in - Table 3. However, this is meant to be descriptive only and readers are cautioned not to compare these very different studies directly. Despite the differences in patient characteristics and outcome assessments, our data are very much in line with most of the outcome data in previous real-world studies, which indicates that apixaban real-world evidence confirms the effectiveness and safety of apixaban in VTE treatment across a spectrum of very different methodologies, somewhat different populations, and outcome event definitions.

\section{Limitations}

There are several limitations to our study, which have been discussed in detail in previous publications. ${ }^{8,19,20}$ For the presented analysis, some specific limitations apply.

\section{Sample Size and Potential for Selection Bias}

Our cohort consisted of 352 patients, and our sample size as well as the small number of outcome events may have been too small to detect clinically relevant differences, which is especially true for our descriptive subgroup analyses. The design of our registry introduces the possibility of a selection bias because local physicians within the network are not instructed on which of their patients should receive which type or dosage of oral anticoagulant therapy.

\section{Lack of Randomized Comparator}

The lack of a direct randomized comparator group needs to be regarded as a limitation. However, many large observational VTE treatment studies exist from the VKA era ${ }^{21}$ and data on VKA complications and VKA treatment discontinuation are well established. ${ }^{22}$ In addition, health record and large claim database analyses have attempted to compare VTE treatment of apixaban with VKA ${ }^{17,23}$ using matching procedures. Although this is certainly an important and valid statistical approach it should be noted that matching will not completely rule out residual confounding, also, because a relevant proportion of patients are often not eligible for matching. Therefore, also the inclusion of a comparator group will cause problems for data analysis and interpretation, as has been demonstrated in the XALIA registry. ${ }^{24,25}$

Despite all potential limitations, in comparison to existing real-world data on apixaban VTE treatment, the long followup duration, the prospective data collection at patient level in daily care patients, and the central adjudication of effectiveness, and safety outcomes are significant advantages of our study.

\section{Conclusion}

In daily care, apixaban treatment for acute VTE is effective and acceptably safe. We found initial apixaban dosing to be in accordance with label in over $90 \%$ of patients and, at 6 and 12 months, persistence was high with low rates of unplanned complete discontinuation. Fatal VTE and fatal bleeding are rare events during apixaban therapy and all-cause mortality is mostly related to underlying diseases, age, or acute conditions. Treatment discontinuation resulted in a relevant increase in VTE recurrence, of which 50\% manifested as PE. Furthermore, bleeding complications were still frequently seen in patients stopping apixaban therapy indicating a selection pattern of stopping anticoagulation mainly in patients at high risk for (anticoagulation unrelated) bleeding despite high risk for VTE recurrence. Consequently, the concept of low dose apixaban therapy in secondary VTE prevention, established in AMPLIFY EXTENSION ${ }^{5}$ needs further evaluation in real-world studies.

\section{What Does This Paper Add? What Is Known About the Subject}

- Treatment of venous thromboembolism (VTE) with apixaban was effective and safe in the AMPLIFY/AMPLIFY EXTENSION trials. However, due to the strict selection criteria in phase III trials, post marketing data are needed to confirm the generalizability of trial data.

- So far, real-world data on apixaban treatment for acute VTE are limited, often derived from retrospective analyses and frequently lacking details on dosing, treatment persistence, or long-term outcomes.

- Especially data for apixaban use in the treatment of patients with VTE provoked by major transient risk factors are needed, since such patients were excluded from participation in AMPLIFY.

\section{What This Paper Adds}

- Data from ongoing, prospective, noninterventional Dresden NOAC Registry aimed to confirm the phase III trial results in daily care and to provide outcome data for up to 2 years.

- In daily care, acute venous thromboembolism (VTE) therapy with apixaban showed acceptably low VTE recurrence and major bleeding rates over a follow-up duration of 2 years. Adherence to dosing recommendations and treatment persistence were satisfactory.

- Apixaban demonstrated little differences in outcome rates for patients with provoked versus unprovoked VTE, confirming a broad generalizability of AMPLIFY trial data.

Ethics

The study protocol of the DRESDEN NOAC REGISTRY was approved by the local ethics committee at the Technical 
University Dresden (AZ EK 349092011) and registered at ClinicalTrials.gov (NCT01588119). The study complies with the principles and requirements of the Declaration of Helsinki. All patients provided written informed consent, including a data protection waiver, before enrolment.

\section{Authors' Contributions}

J.B.-W. has designed this analysis and has written the first draft of the manuscript. L.T., S.M., and C.N. have collected the data. L.T. has performed the statistical analyses and drafted the figures. All authors have provided significant contributions to the written presentation, have critically reviewed and approved the final version of the manuscript.

\section{Funding}

The DRESDEN NOAC REGISTRY is supported by the Gesellschaft für Technologie- und Wissenstransfer der Technischen Universität Dresden (GWT-TUD GmbH), Germany (sponsor), by research funds of the University Hospital "Carl Gustav Carus," Dresden, and by the grants from Bayer HealthCare, Boehringer Ingelheim, Daiichi Sankyo, and Pfizer. All authors declare that these companies and institutions had no influence on the study design, conduct of the study, data collection, statistical analysis, or preparation of the manuscript.

\section{Conflict of Interest}

J.B.-W. has received honoraria and research support from Bayer HealthCare, Boehringer Ingelheim, Daiichi Sankyo, Doasense, BMS/Pfizer, and Portola. S.M. has received honoraria from Bayer HealthCare and Daiichi Sankyo. L. T. received honoraria from Daiichi Sankyo. None of the other authors declared a conflict of interest with regard to the NOAC registry or this manuscript.

\section{Acknowledgments}

We are grateful to all participating patients and physicians, who continue to be maximally supportive in helping to follow patients and provide detailed information/documentation on suspected outcome events.

\section{References}

1 van Es N, Coppens M, Schulman S, Middeldorp S, Büller HR. Direct oral anticoagulants compared with vitamin $\mathrm{K}$ antagonists for acute venous thromboembolism: evidence from phase 3 trials. Blood 2014;124(12):1968-1975

2 Kearon C, Akl EA, Ornelas J, et al. Antithrombotic therapy for VTE disease: Chest guideline and expert panel report. Chest 2016;149 (02):315-352

3 Konstantinides SV, Meyer G, Becattini C, et al; ESC Scientific Document Group. 2019 ESC Guidelines for the diagnosis and management of acute pulmonary embolism developed in collaboration with the European Respiratory Society (ERS). Eur Heart J 2020;41(04):543-603

4 Agnelli G, Buller HR, Cohen A, et al; AMPLIFY Investigators. Oral apixaban for the treatment of acute venous thromboembolism. N Engl J Med 2013;369(09):799-808
5 Agnelli G, Buller HR, Cohen A, et al; AMPLIFY-EXT Investigators. Apixaban for extended treatment of venous thromboembolism. N Engl J Med 2013;368(08):699-708

6 Agnelli G, Becattini C, Meyer G, et al; Caravaggio Investigators. Apixaban for the treatment of venous thromboembolism associated with cancer. N Engl J Med 2020;382(17):1599-1607

7 Beyer-Westendorf J, Lensing AW, Arya R, et al. Choosing wisely: the impact of patient selection on efficacy and safety outcomes in the EINSTEIN-DVT/PE and AMPLIFY trials. Thromb Res 2017; 149:29-37

8 Beyer-Westendorf J, Förster K, Pannach S, et al. Rates, management, and outcome of rivaroxaban bleeding in daily care: results from the Dresden NOAC registry. Blood 2014;124(06): 955-962

9 Schellong SM, Gerlach H, Hach-Wunderle V, et al. Diagnosis of deep-vein thrombosis: adherence to guidelines and outcomes in real-world health care. Thromb Haemost 2009;102(06): 1234-1240

10 Schulman S, Kearon CSubcommittee on Control of Anticoagulation of the Scientific and Standardization Committee of the International Society on Thrombosis and Haemostasis. Definition of major bleeding in clinical investigations of antihemostatic medicinal products in non-surgical patients. J Thromb Haemost 2005;3(04):692-694

11 Helmert S, Marten S, Mizera H, et al. Effectiveness and safety of apixaban therapy in daily-care patients with atrial fibrillation: results from the Dresden NOAC Registry. J Thromb Thrombolysis 2017;44(02):169-178

12 Kearon C, Ageno W, Cannegieter SC, Cosmi B, Geersing GJ, Kyrle PASubcommittees on Control of Anticoagulation, and Predictive and Diagnostic Variables in Thrombotic Disease. Categorization of patients as having provoked or unprovoked venous thromboembolism: guidance from the SSC of ISTH. J Thromb Haemost 2016; 14(07):1480-1483

13 Lutsey PL, Walker RF, MacLehose RF, Alonso A, Adam TJ, Zakai NA. Direct oral anticoagulants and warfarin for venous thromboembolism treatment: trends from 2012 to 2017. Res Pract Thromb Haemost 2019;3(04):668-673

14 Dawwas GK, Brown J, Dietrich E, Park H. Effectiveness and safety of apixaban versus rivaroxaban for prevention of recurrent venous thromboembolism and adverse bleeding events in patients with venous thromboembolism: a retrospective population-based cohort analysis. Lancet Haematol 2019;6(01): e20-e28

15 Dawwas GK, Smith SM, Dietrich E, Lo-Ciganic WH, Park H. Comparative effectiveness and safety of apixaban versus warfarin in patients with venous thromboembolism. Am J Health Syst Pharm 2020;77(03):188-195

16 Haastrup SB, Hellfritzsch M, Rasmussen L, Pottegård A, Grove EL. Use of non-vitamin K antagonist oral anticoagulants 2008-2016: a Danish Nationwide Cohort Study. Basic Clin Pharmacol Toxicol 2018;123(04):452-463

17 Weycker D, Li X, Wygant GD, et al. Effectiveness and safety of apixaban versus warfarin as outpatient treatment of venous thromboembolism in U.S. Clinical Practice. Thromb Haemost 2018;118(11):1951-1961

18 Bott-Kitslaar DM, McBane RD, Casanegra AI, et al. Apixaban and rivaroxaban in patients with acute venous thromboembolism. Mayo Clin Proc 2019;94(07):1242-1252

19 Beyer-Westendorf J, Förster K, Ebertz F, et al. Drug persistence with rivaroxaban therapy in atrial fibrillation patients-results from the Dresden non-interventional oral anticoagulation registry. Europace 2015;17(04):530-538

20 Keller L, Marten S, Hecker J, Sahin K, Tittl L, Beyer-Westendorf J. Venous thromboembolism therapy with rivaroxaban in dailycare patients: results from the Dresden NOAC registry. Int J Cardiol 2018;257:276-282 
21 Willey VJ, Bullano MF, Hauch O, et al. Management patterns and outcomes of patients with venous thromboembolism in the usual community practice setting. Clin Ther 2004;26(07):1149-1159

22 Linkins LA, Choi PT, Douketis JD. Clinical impact of bleeding in patients taking oral anticoagulant therapy for venous thromboembolism: a meta-analysis. Ann Intern Med 2003;139(11):893-900

23 Sindet-Pedersen C, Staerk L, Pallisgaard JL, et al. Safety and effectiveness of rivaroxaban and apixaban in patients with venous thromboembolism: a nationwide study. Eur Heart J Cardiovasc Pharmacother 2018;4(04):220-227
24 Ageno W, Mantovani LG, Haas S, et al. XALIA: rationale and design of a non-interventional study of rivaroxaban compared with standard therapy for initial and long-term anticoagulation in deep vein thrombosis. Thromb J 2014;12:16

25 Ageno W, Mantovani LG, Haas S, et al. Safety and effectiveness of oral rivaroxaban versus standard anticoagulation for the treatment of symptomatic deep-vein thrombosis (XALIA): an international, prospective, non-interventional study. Lancet Haematol 2016;3(01):e12-e21 